\title{
Rechtsgeschichte der Wirtschaft
}

\author{
Seit dem 19. Jahrhundert
}

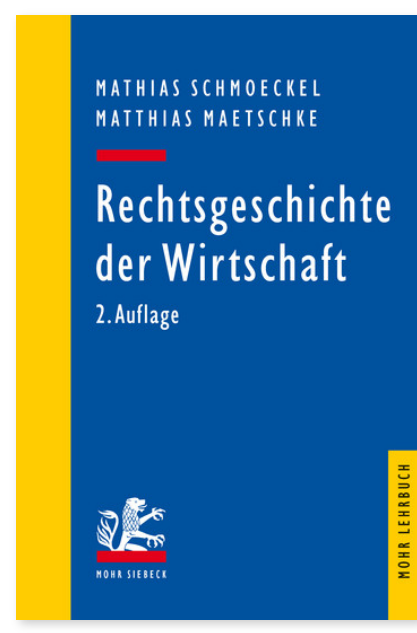

2., überarbeitete und ergänzte Auflage; 2016. XX, 540 Seiten. MLB

ISBN 978-3-16-154505-4 DOI 10.1628/978-3-16-154505-4 eBook PDF $36,00 €$

ISBN 978-3-16-154504-7 Broschur $36,00 €$
Das Werk vermittelt Studierenden der Rechts-, Geschichts- und Wirtschaftswissenschaften einen chronologischen Überblick über die Entwicklung des rechtlichen Rahmens der deutschen Wirtschaft von ca. 1800 an bis heute. Dafür stellt es in verschiedenen Blöcken insbesondere die Entwicklung des Handelsrechts, des gewerblichen Rechtsschutzes, des Gesellschaftsrechts, des Kartellrechts und des Arbeitsrechts dar.

In der Neuauflage sind die entsprechenden Lehrsätze am Ende der Kapitel hinzugekommen. Außerdem werden verschiedene Materien wie Banken und, Versicherungen in einem neuen Kapitel zum Regulierungsrecht behandelt.

"Mathias Schmoeckel ist ein vorzüglich konzipiertes und dokumentiertes Werk zu verdanken, das nicht nur den Studierenden, sondern auch der wissenschaftlichen Arbeit den Zugang zur Entwicklung der Regelung der wirtschaftlichen Abläufe und das Verständnis ihrer komplexen Zusammenhänge wesentlich zu erleichtern vermag.«

Friedrich Kübler Rechtsgeschichte 2009, 215-217

»Hier werden erstmals gut verständlich die Entwicklungen rechtlicher und wirtschaftlicher Schnittpunkte rechtshistorisch gut verständlich dargelegt und somit das Verständnis interdisziplinärer Zusammenhänge gefördert. Allein angesichts der Bewältigung der Aufgabe, ein neues rechtshistorisches Fach zu etablieren und so gut zu strukturieren, muss vor dem Autor der Hut gezogen werden. [...] Es kann daher allen Interessierten uneingeschränkt empfohlen werden.« Christiane Warmbein www.studjur-online.de (18.05.2009)

»Dem Werk ist eine breite Leserschaft zu wünschen, weit über den primär zugrunde gelegten Zweck einer Grundlagenausbildung für den wirtschaftsrechtlichen Schwerpunktbereich hinaus.« Bernd Mertens Zeitschr.f.Neuere Rechtsgeschichte 2010, 320-321

Für Bibliotheken gelten bei diesem Titel abweichende Konditionen; bitte wenden Sie sich an den Vertrieb.

Mathias Schmoeckel Geboren 1963; Studium der Rechtswissenschaft in Bonn, Genf und München; 1993 Promotion; 1999 Habilitation; Geschäftsführender Direktor des Instituts für Deutsche und Rheinische Rechtsgeschichte und Bürgerliches Recht, sowie des Rheinischen Instituts für Notarrecht. https://orcid.org/0000-0003-1504-4228

Matthias Maetschke Geboren 1976; Studium der Rechtswissenschaften an den Universitäten Frankfurt/Main und Bonn; 2006 Promotion; 2008 Zweites juristisches Staatsexamen; Rechtsanwalt im Bereich des privaten Immobilienwirtschaftsrechts in Düsseldorf; seit 2009 Akademischer Rat auf Zeit am Institut für Deutsche und Rheinische Rechtsgeschichte der Universität Bonn; 2015 Erteilung der venia legendi für Bürgerliches Recht, Deutsche Rechtsgeschichte und Historische Rechtsvergleichung.

Jetzt bestellen:

https://mohrsiebeck.com/buch/rechtsgeschichte-der-wirtschaft-9783161545054?no_cache=1 order@mohrsiebeck.com

Telefon: +49 (0)7071-923-17

Telefax: $+49(0) 7071-51104$ 